\title{
Structural properties and enthalpy of formation of magnesium hydride from quantum Monte Carlo calculations
}

\author{
M. Pozzo ${ }^{1}$ and D. Alfè $1,2,3,4$ * \\ ${ }^{1}$ Department of Earth Sciences, \\ University College London, Gower Street, \\ London WC1E 6BT, United Kingdom \\ ${ }^{2}$ Department of Physics and Astronomy, \\ University College London, Gower Street, \\ London WC1E 6BT, United Kingdom \\ ${ }^{3}$ London Centre for Nanotechnology, \\ University College London, 17-19 Gordon Street, \\ London WC1H OAH, United Kingdom \\ ${ }^{4}$ Materials Simulation Laboratory, \\ University College London, Gower Street, \\ London WC1E 6 BT, United Kingdom
}

(Dated: May 31, 2018)

\begin{abstract}
We have used diffusion Monte Carlo (DMC) calculations to study the structural properties of magnesium hydride $\left(\mathrm{MgH}_{2}\right)$, including the pressure-volume equation of state, the cohesive energy and the enthalpy of formation from magnesium bulk and hydrogen gas. The calculations employ pseudopotentials and B-spline basis sets to expand the single particle orbitals used to construct the trial wavefunctions. Extensive tests on system size, time step, and other sources of errors, performed on periodically repeated systems of up to 1050 atoms, show that all these errors together can be reduced to below $10 \mathrm{meV}$ per formula unit. We find excellent agreement with the experiments for the equilibrium volume of both the $\mathrm{Mg}$ and the $\mathrm{MgH}_{2}$ crystals. The cohesive energy of the $\mathrm{Mg}$ crystal is found to be $1.51(1) \mathrm{eV}$, and agrees perfectly with the experimental value of $1.51 \mathrm{eV}$. The enthalpy of formation of $\mathrm{MgH}_{2}$ from $\mathrm{Mg}$ bulk and $\mathrm{H}_{2}$ gas is found to be $0.85 \pm 0.01 \mathrm{eV} /$ formula unit, or $82 \pm 1 \mathrm{~kJ} / \mathrm{mole}$, which is off the experimental one of $76.1 \pm 1 \mathrm{~kJ} / \mathrm{mole}$ only by $6 \mathrm{~kJ} / \mathrm{mole}$. This shows that DMC can almost achieve chemical accuracy $(1 \mathrm{kcal} / \mathrm{mole})$ on this system. Density functional theory errors are shown to be much larger, and depend strongly on the functional employed.
\end{abstract}

\section{INTRODUCTION}

The energetics of metal hydrides has recently become an issue of large scientific and technological interest, mainly because of the revived interest in these materials as potential hydrogen storage media ${ }^{1}$. Magnesium hydride $\left(\mathrm{MgH}_{2}\right)$ is a particularly interesting material, as it can store up to $7.6 \%$ of hydrogen by weight, which is believed to be a large enough quantity for mobile applications, provided that all the hydrogen in the material can be made available when requested, of course. When heated above $\sim 300{ }^{\circ} \mathrm{C}^{2} \mathrm{MgH}_{2}$ decomposes into $\mathrm{Mg}$ bulk and $\mathrm{H}_{2}$ gas, the reaction being endothermic with an enthalpy of decomposition of $76 \mathrm{~kJ} / \mathrm{mole}^{3}$. Conversely, $\mathrm{MgH}_{2}$ can be synthesised by combining $\mathrm{Mg}$ bulk (usually in form of a powder of micro-metre sized grains) and $\mathrm{H}_{2}$ gas. The charging process can take many hours, because of a large energy barrier to dissociate the $\mathrm{H}_{2}$ molecule on the surface of magnesium ${ }^{4}$. As it stands, $\mathrm{MgH}_{2}$ is not considered to be useful for hydrogen storage purposes, because of the high decomposition temperature (ideal decomposition temperature should be in the range $20-100{ }^{\circ} \mathrm{C}$ ), and the slow kinetics of hydrogen intake. A number of attempts are being made to modify this material to improve its properties, including doping it with traces of transition metals 51617 , which have been shown to be very effective at reducing the activation energy for hydrogen dissociation ${ }^{89|10| 11}$, and also somewhat reduce the decomposition temperature of the hydride ${ }^{6}$.

A number of theoretical calculations have been performed on magnesium hydride and related systems (see for example ${ }^{12}$ and references therein; see also $\left.6 / 8|9| 10|11| 13|14| 15 \mid 16\right)$, the most recent ones based on the implementation of quantum mechanics known as density functional theory (DFT) ${ }^{17 / 18}$. Although DFT can often be reliable at predicting trends in the energetics of materials, it can be sometime in error when used to obtain absolute energies. In particular, as we show below, when applied to the calculation of the enthalpy of formation of $\mathrm{MgH}_{2}$, the results are off by as much as $0.3 \mathrm{eV}$ per formula unit, depending on the functional employed, and cohesive energies can be wrong by over $0.5 \mathrm{eV}$.

Quantum Monte Carlo (QMC) techniques $\frac{1920}{120}$ are believed to be one possible way to improve beyond density functional theory. Since they are many order of magnitudes more computationally demanding, the current database of properties of materials calculated with QMC is still rather small, however, the increase in computer power in the past few years is making now possible to perform increasingly more numerous calculations on real systems, and experience is being accumulated on the predictive power of this technique. 
Here we have used QMC to calculate the structural properties of the $\mathrm{Mg}$ and $\mathrm{MgH}_{2}$ crystals, together with their cohesive energies and the enthalpy of formation of $\mathrm{MgH}_{2}$ from $\mathrm{Mg}$ bulk and $\mathrm{H}_{2}$ gas. We find excellent agreement with experiments for the structural properties of the two solids, as well as the cohesive energy of the Mg solid. The enthalpy of formation of $\mathrm{MgH}_{2}$ is slightly overestimated, but the error is of the order of $1 \mathrm{kcal} / \mathrm{mole}$, showing that QMC on this system can almost achieve chemical accuracy.

\section{TECHNIQUES}

\section{A. Density Functional theory calculations}

Density functional theory calculations have been performed with the VAsP code 22 . The interactions between the electrons and the ionic cores was described using the projector augmented method (PAW) 23|24 with the generalised gradient approximations known as $\mathrm{PBE}^{25}$, $\mathrm{PW}^{26}$ or the local density approximation (LDA). The Mg PAW potential has a frozen Ne core and an outermost cutoff radius for the valence orbitals of $1.06 \AA$. The H PAW potential has a cutoff radius of $0.58 \AA$. Single particle orbitals were expanded in plane waves with a plane-wave cutoff of 270 eV, and a cutoff of $1600 \mathrm{eV}$ was used for the charge density. Such a large cutoff in the charge density (4 times larger than the typical one used by default) is necessary to obtain very accurate forces which are used to calculate the vibrational properties of the crystals. Calculations were performed by requiring a self-consistency convergence on the total energy of $10^{-8} \mathrm{eV}$ per simulation cell. With these prescriptions convergence on the forces was at worse equal to $0.2 \mathrm{meV} / \AA$, and one or two order of magnitudes smaller for most atoms in the simulation cell. Brillouin Zone integration was performed using k-point sampling, with 18x18x12 and 10x10x15 Monkhorst-Pack 27 grids on the Mg and $\mathrm{MgH}_{2}$ primitive cells respectively. With these densities of $\mathbf{k}$-points the structural parameters are converged to better than $0.1 \%$, and the total energies to better than $1 \mathrm{meV} /$ primitive cell.

\section{B. Quantum Monte Carlo calculations}

Quantum Monte Carlo techniques have being extensively described elsewhere ${ }^{19 \mid 20}$, so here we only report the main technical details used in this work. Calculations have been performed using the CASINO code ${ }^{21}$. Diffusion Monte Carlo calculations have been performed using trial wavefunctions of the Slater-Jatrow type:

$$
\Psi_{T}(\mathbf{R})=D^{\uparrow} D^{\downarrow} e^{J}
$$

where $D^{\uparrow}$ and $D^{\downarrow}$ are Slater determinants of up- and down-spin single-electron orbitals, and $e^{J}$ is the so called Jastrow factor, which is the exponential of a sum of one-body (electron-nucleus), two-body (electron-electron) and three body (electron-electron-nucleus) terms, which are parametrised functions of electron-nucleus, electron-electron and electron-electron-nucleus separations, and are designed to satisfy the cusp conditions. The parameters in the Jastrow factor are varied to minimise the variance of the local energy $E_{L}(\mathbf{R}) \equiv \Psi_{T}^{-1}(\mathbf{R}) \hat{H} \Psi_{T}(\mathbf{R})$.

Imaginary time evolution of the Schrödinger equation has been performed with the usual short time approximation, and the locality approximation 28 . Time step errors have been carefully analysed later in the paper. Since the locality approximation introduces an uncontrollable error with respect to which the DMC energy is non-variational, we also tested the scheme of Casula 29 , which treats the non local part of the pseudopotential in a consistent variational scheme. We found that the zero time step extrapolation of the energies in the two scheme differed very little, which suggests that the errors in either case is rather small30. However, we also found that the time step error is much smaller in the locality approximation in this particular case (this may not be true in general for other systems), and therefore we decided to use the locality approximation throughout the work which allowed us to work with a larger time step.

We used Dirac-Fock pseudopotentials $(\mathrm{PP})$ for $\mathrm{Mg}$ and $\mathrm{H}^{31}$. The $\mathrm{Mg} \mathrm{PP}$ has a frozen Ne core and a core radius of $1.43 \AA$, the H PP has a core radius of $0.26 \AA$. The single particle orbitals have been obtained by DFT plane-wave (PW) calculations using the LDA and a PW cutoff of $3400 \mathrm{eV}$, using the PWscF package ${ }^{32}$. Such a large PW cutoff is due to the very small H PP core radius, and was found to be necessary to reduce the variance of the local energy as much as possible. We then exploited the approximate equivalence between PW and B-splines 33 to expand the single particle orbitals in a basis of B-spline, as described in Ref. ${ }^{34}$, using the natural B-spline grid spacing given by $a=\pi / G_{\max }$, where $G_{\max }$ is the length of the largest vector employed in the PW calculations.

We used a diffusion Monte Carlo time step of 0.05 a.u., which was found to result in errors of about $2 \mathrm{meV} / \mathrm{f}$.u. (see below). With this time step the acceptance ratios were 99.2 and $99.7 \%$ for the $\mathrm{MgH}_{2}$ and $\mathrm{Mg}$ crystals respectively. Total energies in the solids were obtained by correcting the raw DMC data with DFT-LDA calculations performed on 
the same cell size but a fully converged Brillouin zone sampling, and then extrapolating these corrected DMC data to infinite size (see below). The DMC calculations were performed using the Ewald interaction to model electron-electron interactions. The number of walkers in the DMC simulations varied with the size of the systems, and was never less than 1280 .

\section{RESULTS}

$\mathrm{Mg}$ bulk has the hexagonal close packed structure, which is specified by a lattice parameter $a$ and the ratio $c / a$ of the vertical axis to one of the horizontal ones. The primitive cell contains two atoms, one at the origin and the other at $(1 / 3,2 / 3,0.5)$ in lattice vectors units. The $\mathrm{MgH}_{2}$ solid has a tetragonal structure of rutile type (see Fig. 1), specified by a lattice parameter $a$ and the $c / a$ ratio. The primitive cell has two $\mathrm{Mg}$ atoms, one at the origin and the other in the centre of the cell at $(1 / 2,1 / 2,1 / 2)$ plus four hydrogen atoms at $( \pm x, \pm x, 0)$ and $(1 / 2 \pm x, 1 / 2 \mp x, 1 / 2)$. The exact values of $c / a$ and $\mathrm{x}$ depend on pressure, and at ambient conditions are found to be $c / a=0.6687$ and $\mathrm{x}=0.304^{35}$.

\section{A. Zero point energies and high temperature vibrational effects}

In order to compare the calculated structural parameters and cohesive energies with the experimental ones we need to study the vibrational properties of the crystals. This is because the experimental parameters are usually determined at ambient conditions, and room temperature thermal expansion for the $\mathrm{Mg}_{\mathrm{g} d} \mathrm{MgH}_{2}$ solids is likely to be significant.

We studied these vibrational properties within the quasi-harmonic approximation, which far from the melting temperatures provides accurate enough results for the thermal expansion of solids. This is certainly the case for the $\mathrm{Mg}$ and $\mathrm{MgH}_{2}$ solids at room temperature.

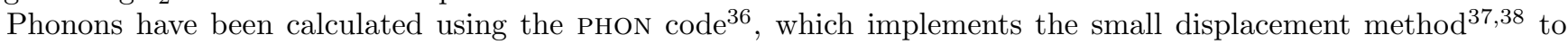
obtain the force constant matrix in crystals. The methods exploits the linearity relation between the displacement of the atoms from their equilibrium positions and the forces induced on all the atoms in the crystal, which holds in the harmonic approximation for small enough displacements. The method is applied by constructing a supercell which is a multiple of the primitive cell in the three spacial directions, then the atoms in the primitive cell are displaced by small amounts along three linearly independent directions and the forces induced on all the atoms in the supercell are used to construct the force constant matrix. Symmetries can usually be used to reduce the total number of displacements needed, and also to symmetrise the force constant matrix ${ }^{37}$. For bulk $\mathrm{Mg}$, which has the hexagonal closed packed crystal structure, only two displacements are needed, one in the basal plane and one orthogonal to it (in fact, one single off symmetry displacements would be sufficient, although this would break the symmetry of the supercell and require a larger number of $\mathbf{k}$-points in the DFT calculation of forces). $\mathrm{MgH}_{2}$ has the tetragonal structure of rutile $\mathrm{TiO}_{2}$, with two $\mathrm{Mg}$ and four $\mathrm{H}$ atoms in the primitive cell, and the total number of displacements needed in this case is 4 (one could reduce the total number of displacements to 2 by sacrificing symmetries). If the supercell is large enough so that the forces on the atoms sitting near the edges are small, then the calculated force constant matrix becomes a good approximation of the exact one. Magnesium bulk is a metal, and convergence of the force constant matrix with the size of the supercell is readily achieved: we found that with cell containing 36 atoms (3x3x2) the $\mathrm{ZPE}$ is converged to within $0.1 \mathrm{meV} /$ atom (tested using supercells containing up to 150 atoms). However, $\mathrm{MgH}_{2}$ is an insulator, and long range Coulomb interactions make convergence slower. Nevertheless, we found that already by using a cell containing 72 atoms $(2 \times 2 \times 3$ supercell) the ZPE can be calculated with an accuracy of 0.5 meV/fu (tests used supercells containing up to 576 atoms). All calculations were performed with DFT-PBE.

Phonons calculated with the direct method described above may suffer from inaccuracies due to the size of the displacements and/or numerical noise in the calculated forces. To reduce the latter, one would like to maximise the size of the displacements, but too large displacements would cause departure from the harmonic regime. A compromise between these two opposite requirements then needs to be found, and this is usually achieved with displacement sizes of the order of a fraction of a percent of the inter-atomic distances. In order to test the size of the displacements we repeated the calculations using displacements of $0.067 \AA, 0.04 \AA, 0.02 \AA$, and $0.01 \AA$, and we found that even with the largest displacement the $\mathrm{ZPE}$ energy is converged to less than $0.2 \mathrm{meV} /$ atom in $\mathrm{Mg}$ and $1 \mathrm{meV} / \mathrm{fu}$ in $\mathrm{MgH}$. We then decided to use displacements of $0.04 \AA$.

The fundamental vibrational frequency of the $\mathrm{H}_{2}$ molecule has been obtained by calculating the total energy of the $\mathrm{H}_{2}$ molecule in a large cubic box of size $13.5 \AA$ for 5 different values of the $\mathrm{H}-\mathrm{H}$ distance, ranging from $R_{0}-0.0135 \AA$ to $R_{0}+0.0135 \AA$, where $R_{0}=0.75 \AA$ is the calculated equilibrium distance with DFT-PBE. The 5 energies have been fitted to a parabola, providing a force constant of $33.35 \mathrm{eV} / \AA^{2}$ which corresponds to a stretching vibrational frequency of $127 \mathrm{THz}$ (only slightly lower that the experimental value of $131.8 \mathrm{THz}$ ), giving a ZPE of $0.263 \mathrm{eV}$. 
TABLE I: Bulk properties (Volume/fu $\mathrm{V}_{0}$ in $\AA^{3}$, and bulk modulus $\mathrm{k}_{0}$ in $\mathrm{GPa}$ ) and cohesive energies $\left(\mathrm{E}_{\text {coh }}\right.$, in eV) of $\mathrm{Mg}$ and $\mathrm{MgH}_{2}$. Calculated properties are reported at zero temperature with and without zero point energies (ZPE) and at the temperatures at which the experimental data have been taken. Also reported is the binding energy of the $\mathrm{H}_{2}$ molecule

\begin{tabular}{|c|c|c|c|c|c|}
\hline & & \multicolumn{3}{|c|}{$\mathrm{T}=0 \mathrm{~K}$} & \multirow{2}{*}{$\frac{\mathrm{T}^{a}}{\mathrm{~V}_{0}, \mathrm{k}_{0}}$} \\
\hline & & $\begin{array}{c}\mathrm{V}_{0}, \mathrm{k}_{0} \\
\text { (no ZPE) }\end{array}$ & $\begin{array}{c}\mathrm{V}_{0}, \mathrm{k}_{0} \\
\text { (with } \mathrm{ZPE} \text { ) }\end{array}$ & $\begin{array}{c}\mathrm{E}_{c o h} \\
\text { (with ZPE) }\end{array}$ & \\
\hline \multicolumn{6}{|l|}{$\mathrm{Mg}$} \\
\hline & LDA & $21.59,40.6$ & $21.80,39.3$ & -1.74 & $22.14,36.4$ \\
\hline & PBE & $22.86,36.5$ & $23.08,35.9$ & -1.47 & $23.47,34.0$ \\
\hline & PW91 & $22.86,36.4$ & $23.10,35.2$ & -1.45 & $23.50,32.6$ \\
\hline & {$[$ Exp.] } & & & {$[-1.51]^{b}$} & {$\left[23.24^{c}, 36.8 \pm 3.0^{d}\right.$} \\
\hline & $\mathrm{DMC}$ & $22.96 \pm 0.05,35.5 \pm 1.2$ & $23.19 \pm 0.05,34.4 \pm 1.4$ & $-1.51 \pm 0.01$ & $23.61 \pm 0.04,31.2 \pm 2.4$ \\
\hline \multicolumn{6}{|c|}{$\mathrm{MgH}_{2}$} \\
\hline & LDA & $29.36,55.5$ & $30.32,51.4$ & -7.16 & $30.36,49.9$ \\
\hline & $\mathrm{PBE}$ & $30.84,51.1$ & $31.92,45.8$ & -6.17 & $32.03,43.5$ \\
\hline & PW91 & $30.72,51.5$ & $31.79,46.4$ & -6.27 & $31.89,43.9$ \\
\hline & [Exp.] & & & {$\left[-6.78 \pm 0.01^{e}\right]$} & {$\left[30.49^{f},-\right]$} \\
\hline & $\mathrm{DMC}$ & $29.48 \pm 0.03,58.6 \pm 3.6$ & $30.53 \pm 0.05,42.0 \pm 1.5$ & $-6.84 \pm 0.01$ & $30.58 \pm 0.06,39.5 \pm 1.7$ \\
\hline \multicolumn{6}{|l|}{$\mathrm{H}_{2}$} \\
\hline & LDA & & & -4.59 & \\
\hline & PBE & & & -4.23 & \\
\hline & PW91 & & & -4.25 & \\
\hline & {$[\mathrm{Exp}]$} & & & {$\left[-4.48^{g}\right]$} & \\
\hline & $\mathrm{DMC}$ & & & $-4.484 \pm 0.002$ & \\
\hline
\end{tabular}

${ }^{a} \mathrm{~T}=298 \mathrm{~K}$ for $\mathrm{Mg}, \mathrm{T}=260 \mathrm{~K}$ for $\mathrm{MgH}_{2}$.

${ }^{b}$ Ref. 41

${ }^{c}$ Ref. 42

${ }^{d}$ Ref. 43

${ }^{e}$ Ref. 3 ,

${ }^{f}$ Ref. 35

${ }^{g}$ Ref. 39

\section{B. Density functional theory results}

Initially, we performed DFT calculations on the crystals with PBE, PW91 and LDA. Energy versus volume curves were fitted to a Birch-Murnaghan equation of state ${ }^{40}$, which provided equilibrium volumes and bulk moduli. In the range of volumes considered, $c / a$ 's do not change very much from their zero pressure values, and the structural parameters are essentially unchanged if $c / a$ is kept fixed. Therefore, for simplicity we decided to fix $c / a$ to their calculated zero pressure values of 1.621 and 0.6682 for $\mathrm{Mg}$ and $\mathrm{MgH}_{2}$ respectively. The $\mathrm{MgH}_{2}$ crystal has an additional degree of freedom, which defines the position of the $\mathrm{H}$ atoms in the lattice. This has also been optimised by fully relaxing the crystal at each different volume. These relaxations are essential in the calculation of phonons, because if the crystal is not in its ground state imaginary phonon frequencies appear. However, as far as the energy is concerned, the differences from calculations in which the $\mathrm{H}$ positions are kept at their zero pressure equilibrium values are undetectable.

In Table [1 we report the structural parameters of $\mathrm{Mg}$ and $\mathrm{MgH}_{2}$ calculated with the three density functionals, and we report the results both at zero temperature (with and without $\mathrm{ZPE}$ ) and at room temperature. Both $\mathrm{Mg}$ and $\mathrm{MgH}_{2}$ are fairly soft materials, with bulk moduli of the order of 40 and 50 GPa. Room temperature thermal pressure are about 1 and $1.8 \mathrm{GPa}$ for $\mathrm{Mg}$ and $\mathrm{MgH}_{2}$ respectively, which means volume thermal expansion is about $2 \%$ and $3.5 \%$ for the two solids. This is significant, and cannot be ignored in a fair comparison with the experimental data. We also report in the same table the cohesive energies of the two solids. The experimental cohesive energy of $\mathrm{MgH}_{2}$ can be estimated by combining the cohesive energy of the $\mathrm{Mg}$ crystal (1.51 eV/atom), the dissociation energy of the hydrogen molecule (4.48 eV/molecule) and the enthalpy of formation of $\mathrm{MgH}_{2}$ from $\mathrm{Mg}$ and $\mathrm{H}_{2}$, whose value extrapolated at zero temperature is $0.79 \pm 0.01 \mathrm{eV} / \mathrm{fu}^{3}$, which therefore give a result of $6.78 \pm 0.01 \mathrm{eV} / \mathrm{fu}$. By comparing the calculated cohesive energies with the experimental ones it is clear that the three functionals provide quite scattered results, with the LDA doing better on $\mathrm{MgH} 2$ and $\mathrm{PBE}$ doing better on $\mathrm{Mg}$. It is also apparent that errors can be significant, of up $0.6 \mathrm{eV}$ for PBE. This error is well over 10 times a kcal/mole, which is the typical quantity cited as chemical accuracy. 


\section{Diffusion Monte Carlo results}

\section{Time step tests}

The dependence of the DMC energy on time step in the $\mathrm{MgH}_{2}$ crystal was studied by repeating simulations with a $2 \times 2 \times 3$ supercell (72 atoms) at time steps ranging from 0.005 to 0.15 a.u.. Calculations were performed at the volume of $30.835 \AA^{3} / \mathrm{fu}$, and using the $A$ point $(0.5,0.5,0.5)$ which is at one corner of the Brillouin zone. For the Mg crystal we used a $3 \times 3 \times 2$ supercell (36 atoms), a volume of $22.785 \AA^{3} /$ atom and the $\mathrm{H}$ point $(0.5,0.5,0.5)$, also at one corner of the Brillouin zone.

Results of total energy/fu for $\mathrm{MgH}_{2}$ and total energy/atom for $\mathrm{Mg}$ are displayed in Fig. 2, from which it is evident that using a time step of $0.05 \mathrm{a}$.u. time step errors are well below $5 \mathrm{meV} / \mathrm{fu}$. In Fig. 2 we also display the results obtained with the scheme proposed by Casula ${ }^{29}$, and we observe that for short enough time steps the two sets of energies are very close, and extrapolate to roughly the same value in the limit of zero time step (to less than 5 $\mathrm{meV} / \mathrm{fu})$. As mentioned earlier, this suggests that the error introduced with either scheme is very small. However, the locality approximation results in a much weaker dependence of the DMC energy on time step and this is what we used because it allowed us to work with much larger time steps. We note that for the Mg crystal the time step error is much smaller, which in principle would allow us to work with larger time steps, however, for consistency, we used the same time step of 0.05 a.u. also for the $\mathrm{Mg}$ crystal.

To calculate the total energies of the $\mathrm{Mg}$ atom and the $\mathrm{H}_{2}$ molecule we used trial wavefunctions obtained from plane wave calculations in which the $\mathrm{Mg}$ atom or the $\mathrm{H}_{2}$ molecule was placed at the centre of a large cubic box with a side of $13.5 \AA$. The DMC calculations were then performed using B-splines and no periodically boundary conditions. We display in Fig. 3 the DMC energies as function of time step, from which we can obtain very accurate zero time step values. In the case of $\mathrm{Mg}$ we also performed one calculation with the scheme of Casula 29 , which gave essentially the same energy. For the $\mathrm{H}_{2}$ molecule we display the binding energy calculated at the equilibrium distance of $0.75 \AA$, obtained by subtracting from the energy of the molecule twice the energy of the $\mathrm{H}$ atom, which is calculated to be 13.60635(5) eV. Both the energies of the $\mathrm{H}$ atom and the $\mathrm{H}$ molecule are in excellent agreement with the experimental data.

\section{The Mg crystal}

In the Mg crystal we studied the dependence of the DMC energy on the size of the simulation cell by repeating the calculations with $4 \times 4 \times 3,5 \times 5 \times 3,6 \times 6 \times 4,8 \times 8 \times 5$ and 9x9x6 supercells, containing 96, 150, 288, 640 and 972 atoms respectively. Results are displayed in Fig. 4. where we show the total energies/atom $E_{N}$ as function of $1 / N$, with $\mathrm{N}$ the number of atoms in the simulation cell. On the same graph we also show the energies $E_{N}^{c}=E_{N}+\left[E_{\infty}^{D F T}-E_{N}^{D F T}\right]$, where $E_{\infty}^{D F T}$ are the DFT energies calculated with fully converged k-point sampling, and $E_{N}^{D F T}$ are the DFT energies calculated with k-point samplings corresponding to the $N$-atom cells used in the DMC calculations. It is clear that the raw DMC energies $E_{N}$ are quite scattered and somewhat difficult to extrapolate to infinite size. This is due to the metallic nature of $\mathrm{Mg}$. However, the DFT corrected energies $E_{N}^{c}$ are much better behaved, with data fitting quite well onto a straight line, which makes it possible to extrapolate to infinite size. In particular, we note that with no loss of accuracy we can also use only the calculations with the $4 \times 4 \times 3,5 \times 5 \times 3$ and $6 \times 6 \times 4$ supercells to extrapolate to essentially the same infinite size value.

The calculations with these three supercell sizes were then repeated at 8 different volumes, between 21 and $25 \AA^{3}$ /atom. At each volume the DFT corrected DMC results were extrapolated to infinite size and the results were fitted to a Birch-Murnaghan equation of state to obtain the structural parameters. We performed the fit by weighing each energy point $E_{i}$ point with $1 / \sigma_{i}^{2}$, where $\sigma_{i}$ is the standard error on $E_{i}$. We report in Table I the results obtained both at zero temperature (with and without zero point energy) and at room temperature. The latter are also shown in Fig. 5. The room temperature corrected DMC results slightly overestimate the equilibrium volume, and also underestimate the bulk modulus, but the calculated cohesive energy is in perfect agreement with the experimental data.

\section{The $\mathrm{MgH}_{2}$ crystal}

For the $\mathrm{MgH}_{2}$ crystal size effects were studied using $2 \times 2 \times 3,3 \times 3 \times 4,4 \times 4 \times 6$ and $5 \times 5 \times 7$ supercells, containing 72,216 , 576 and 1050 atoms respectively. These tests were performed at the volume of $30 \AA^{3} /$ fu. The results for the four sizes studied are displayed in Fig. 6. where we show total energies/fu $E_{N}$ as function of $1 / N$, as well as the DFT corrected 
energies $E_{N}^{c}$. In this case the DFT corrections are much smaller, which is not surprising because of the large band gap in $\mathrm{MgH}_{2}$. A small difference between the two sets of data can be observed for the smallest sizes, but it is clear that they both fit very well onto straight lines, which allows us to easily extrapolate the results to infinite size. In fact, in this case the extrapolated results for the two sets only differ by $5 \mathrm{meV} /$ atom.

The calculations were repeated at 7 different volumes between 28 and $32.5 \AA^{3} / \mathrm{fu}$, and the DFT corrected DMC results were then fitted with a Birch-Murnaghan equation of state to obtain the structural parameters. Also in this case we used the inverse of the variances to weigh each point in the fit. We report in Table I the results obtained both at zero temperature (with and without zero point energy) and at $\mathrm{T}=260 \mathrm{~K}$, which is the temperature at which the experimental data are reported ${ }^{35}$. The high temperature results are also shown in Fig. 7. It is clear that once thermal effects are added onto the calculations the agreement with the experimental equilibrium volume is in almost perfect agreement. The cohesive energy is slightly overestimated, but the error is only $0.06 \mathrm{eV}$, i.e. of the order of chemical accuracy.

\section{Enthalpy of formation of $\mathrm{MgH}_{2}$}

We can now calculate the enthalpy of formation of $\mathrm{MgH}_{2}$ from $\mathrm{Mg}$ bulk and $\mathrm{H}_{2}$ in the gas phase by adding the cohesive energies of the $\mathrm{MgH}_{2}$ and $\mathrm{Mg}$ crystals to the binding energy of the $\mathrm{H}_{2}$ molecule. We obtain enthalpy of formations of $0.82,0.47$ and $0.57 \mathrm{eV} / \mathrm{fu}$ with $\mathrm{LDA}, \mathrm{PBE}$ and PW91 respectively, and with DMC we obtain the value $0.85 \pm 0.01 \mathrm{eV} / \mathrm{fu}$. The LDA value is very accurate, but this is the result of large cancellations of errors in the cohesive energies of the crystals and the binding energy of the $\mathrm{H}_{2}$ molecule. The DMC result is only $0.06 \mathrm{eV}$ higher than the experimental value of $0.79 \pm 0.01 \mathrm{eV} / \mathrm{fu}$, however in this case both the cohesive energies of the crystals and the binding energy of the $\mathrm{H}_{2}$ molecule are very accurate.

\section{CONCLUSIONS}

We pointed out in this work the difficulty of using density functional theory to calculate the enthalpy of formation of $\mathrm{MgH}_{2}$ with high accuracy. We studied the effect of three different exchange-correlation functionals, PW91, PBE and LDA, and found that although the GGA ones appear to work better on the Mg solid, the LDA gives better results on the $\mathrm{MgH}_{2}$ solid. It turns out, therefore, that is difficult to get a good DFT value for the enthalpy of formation of $\mathrm{MgH}_{2}$ : the two GGA functionals give an enthalpy of formation in error of more than 0.2 and $0.3 \mathrm{eV} / \mathrm{fu}$ respectively. The LDA is the functional that does best, but for the wrong reason, because the cohesive energies of the crystals and the binding energies of the molecule are wrong by up to $0.4 \mathrm{eV} / \mathrm{fu}$, and the enthalpy of formation is accurate only because of large cancellation of errors.

Diffusion Monte Carlo appears to deliver much better accuracy in general. We have shown that the DMC equilibrium volumes of $\mathrm{MgH}_{2}$ agrees perfectly with the experimental one, once high temperature thermal expansion is included in the calculations, and the equilibrium volume of $\mathrm{Mg}$ is only slightly overestimated. The cohesive energy of $\mathrm{Mg}$ is also predicted in perfect agreement with the experimental datum, and so is the binding energy of the $\mathrm{H}_{2}$ molecule. A small error is present in the cohesive energy of the $\mathrm{MgH}_{2}$ crystal, which determines the small inaccuracy in the enthalpy of formation, for which we find a DMC value of $0.85 \pm 0.01 \mathrm{eV} / \mathrm{fu}$. However, this is only 0.06 higher than the accepted experimental one of $0.79 \pm 0.01 \mathrm{eV} / \mathrm{fu}$, or $76.1 \pm 1 \mathrm{~kJ} / \mathrm{mole}$. This result is not very far from the LDA value, but with the important difference that now all three terms that enter the enthalpy of formation are calculated accurately, and we don't rely on fortunate cancellation of errors.

Although the DMC error is slightly larger than $1 \mathrm{kcal} /$ mole, and therefore we cannot claim chemical accuracy, we are not far from it, and therefore we argue that quantum Monte Carlo techniques have useful predictive power in the search of metal hydrides with workable decomposition temperatures.

\section{Acknowledgments}

The computations were performed on the HPCx service, using allocations of time from NERC, and on HECToR. Calculations have also been performed on the LCN cluster at University College London. This work was conducted 
as part of a EURYI scheme award as provided by EPSRC (see www.esf.org/euryi).

* Electronic address: d.alfe@ucl.ac.uk

${ }^{1}$ L. Schlapbach and A. Züttel, Nature (London) 414, 353 (2001).

2 B. Bogdanovic, K. Bohmhammel, B. Christ, A. Reiser, K. Schlichte, R. Vehlen and U. Wolf, J. Alloys Comp. 282, 84 (1999).

3 M. Yamaguchi and E. Akiba, in Material Science and Technology, vol. 3B, edited by R. W. Cahn, P. Haasen and E. J. Kramer (New York: VCH, 1994), p. 333.

${ }^{4}$ P. T. Sprunger and E. W. Plummer, Chem. Phys. Lett. 187, 559 (1991).

${ }^{5}$ G. Liang, J. Huot, S. Boily, A. Van Neste and R. Schulz, J. Alloys Compd. 292, 247 (1999).

${ }^{6}$ C. X. Shang, M. Bououdina, Y. Song and Z. X. Guo, Int. J. Hydrogen Energy 29, 73 (2004).

7 N. Hanada, T. Ichigawa and H. Fujii, J. Phys. Chem. B 109, 7188 (2005).

8 A. J. Du, S. C. Smith, X. D. Yao, and G. Q. Lu, J. Phys. Chem. B 109, 18037 (2005).

9 A. J. Du, S. C. Smith, X. D. Yao, and G. Q. Lu, J. Phys. Chem. B 110, 21747 (2006).

10 A. J. Du, S. C. Smith, X. D. Yao, and G. Q. Lu, J. Am. Chem. Soc. 129, 10201 (2007).

11 M. Pozzo, D. Alfè, A. Amieiro, S. French and A. Pratt, unpublished.

12 R. Yu and P. K. Lam, Phys. Rev. B 37, 8730 (1988).

13 P. Vajeeston, P. Ravindran, A. Kjekshus, and H. Fjellvåg, Phys. Rev. Lett. 89, 175506 (2002).

14 Y. Song, Z. X. Guo and R. Yang, Phys. Rev. B 69, 094205 (2004).

15 M. J. van Setten, G. A. de Wijs, V. A. Popa, and G. Brocks, Phys. Rev. B 72, 073107 (2005).

16 T. Vegge, L. S. Hedegaard-Jensen, J. Bonde, T. R. Munter and J. K. Nørskov, J. Alloys Compd. 386, 1 (2005).

17 P. Hohenberg, W. Kohn, Phys. Rev. B 136, 864 (1964).

18 W. Kohn and L.J. Sham. Phys. Rev. A 140, 1133 (1965).

19 W. M. C. Foulkes, L. Mitáš, R. J. Needs, and G. Rajagopal, Rev. Mod. Phys. 73, 33 (2001).

20 C. J. Umrigar, M. P. Nightingale, K. J. Runge, J. Chem. Phys., 99, 2865 (1993).

21 R.J. Needs, M.D. Towler, N.D. Drummond and P. Lopez Rios, CASINO version 2.1 User Manual, University of Cambridge, Cambridge (2007).

${ }^{22}$ G. Kresse and J. Furthmuller, Comput. Mater. Sci. 6 (1996) 15; Phys. Rev. B 54, 11169 (1996).

${ }^{23}$ P. E. Blöchl, Phys. Rev. B, 50, 17953 (1994).

${ }^{24}$ G. Kresse and D. Joubert, Phys. Rev. B, 59, 1758 (1999).

25 J.P. Perdew, K. Burke and M. Ernzerhof. Phys. Rev. Lett. 77, 3865 (1996).

26 J.P. Perdew and Y. Wang. Phys. Rev. B 45, 13244 (1992).

27 H. J. Monkhorst and J. D. Pack, Phys. Rev. B 13, 5188 (1976).

28 L. Mitáš, E. L. Shirley, D. M. Ceperley, J. Chem. Phys. 95, 3467 (1991).

29 M. Casula, Phys. Rev. B 74, 161102 (2006).

${ }^{30}$ Of course, it is possible that the Casula scheme induces a large positive error in the DMC energy, and that by chance the error induced by the locality approximation is also positive and of the same size. However, since the two scheme are completely different, we believe that this is unlikely.

31 J. R. Trail and R. J. Needs, J. Chem. Phys. 122, 014112 (2005); ibid J. Chem. Phys. 122, 174109 (2005), see also www.tcm.phy.cam.ac.uk/ mdt26/casino2_pseudopotentials.html.

32 S. Baroni, A. Dal Corso, S. de Gironcoli, and P. Giannozzi, http://www.pwscf.org

33 E. Hernández, M. J. Gillan and C. M. Goringe, Phys. Rev. B 55, 13485 (1997).

34 D. Alfè and M. J. Gillan, Phys. Rev B, 70, 161101(R) (2004).

35 M. Bortz, B. Bertheville, G. Böttger, K. Yvon, J. Alloys Compd. 287, L4 (1999)

36 D. Alfè, (1998). Program available at http://chianti.geol.ucl.ac.uk/ dario, D. Alfè, G. D. Price, M. J. Gillan, Phys. Rev. B, 64, 04512316 (2001).

37 G. Kresse, J. Furthmüller and J. Hafner, Europhys. Lett. 32, 729 (1995).

38 D. Alfè, G. D. Price, M. J. Gillan, Phys. Rev. B, 64, 045123 (2001).

39 B. H. Bransden and C. J. Joachin, Physics of Atoms and Molecules (Wiley, New York, 1983).

40 F. Birch, Phys. Rev. 71, 809 (1947).

41 C. Kittel, Introduction to Solid State Physics, 7th ed. (Wiley, New York, 1996).

42 http://www.webelements.com

43 D. Errandonea, Y. Meng, D. Häusermann, and T. Uchida, J. Phys.: Condens. Matter 15, 1277 (2003). 


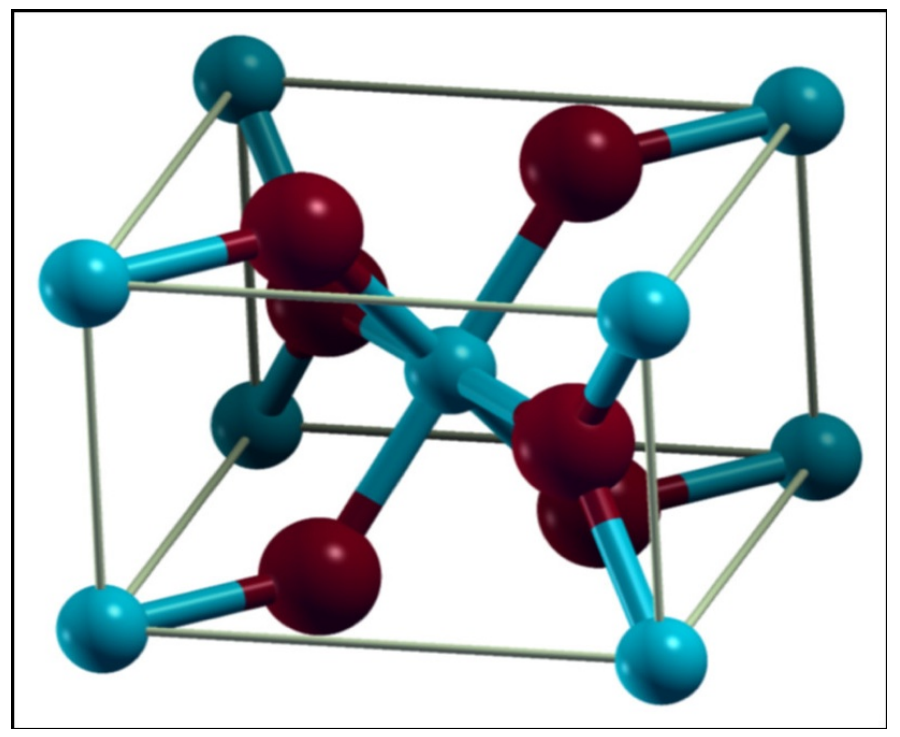

FIG. 1: (colour) Crystal structure of $\mathrm{MgH}_{2}$ (see also text). The $\mathrm{Mg}$ and $\mathrm{H}$ atoms are represented respectively by light blue and dark red colours.
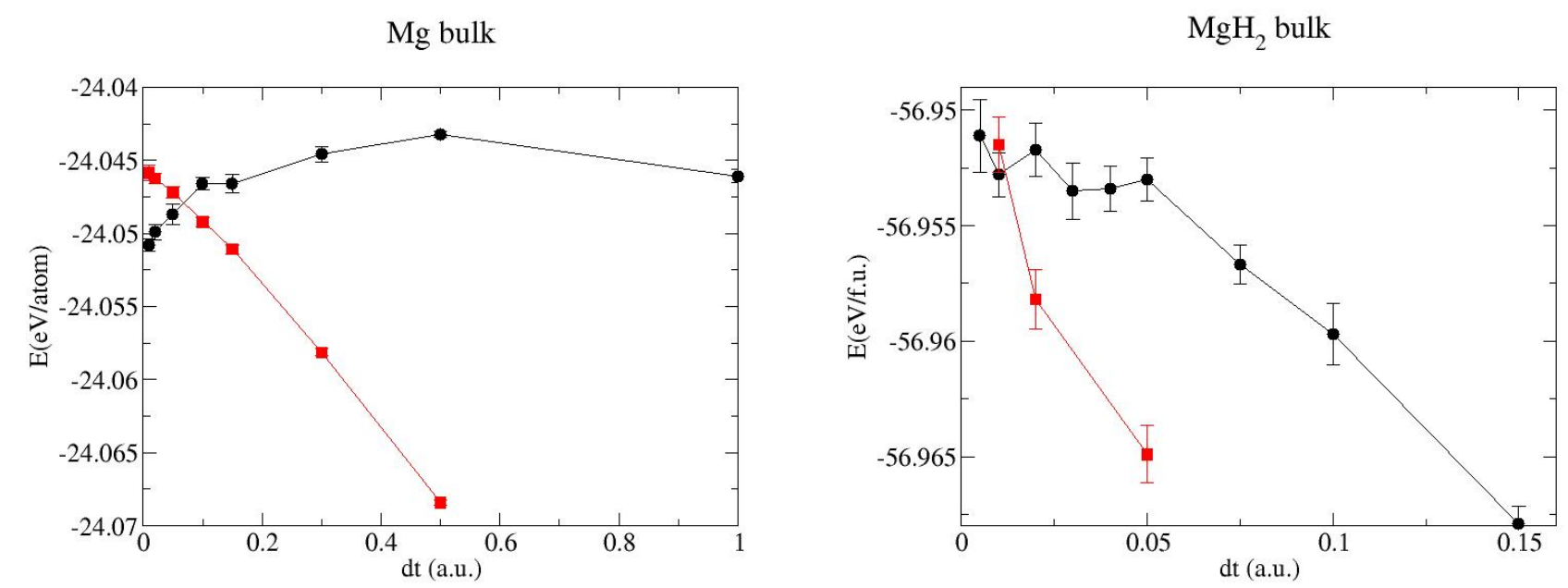

FIG. 2: Diffusion Monte Carlo energies for $\mathrm{Mg}$ bulk (left panel) and $\mathrm{MgH}_{2}$ bulk (right panel) as function of time step. Dots and squares correspond to calculations performed with the locality approximation and with the scheme proposed by Casula respectively. 
$\mathrm{Mg}$ atom

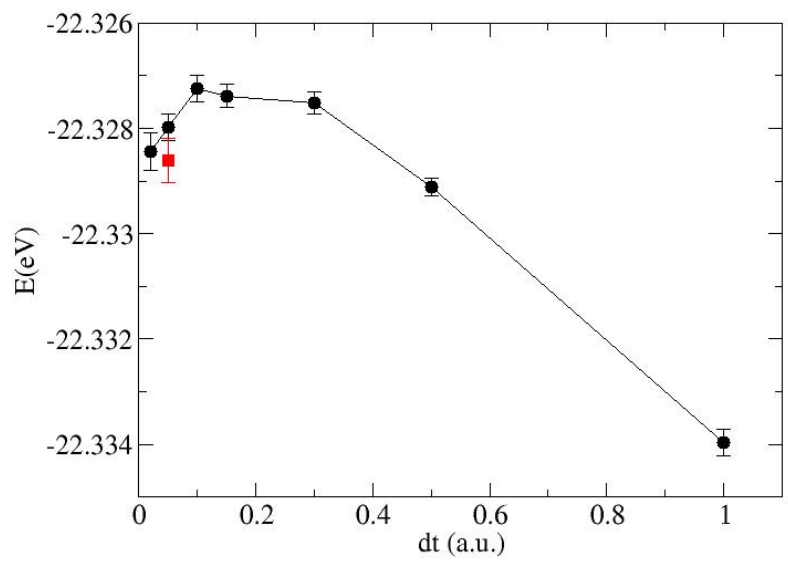

$\mathrm{H}_{2}$ molecule

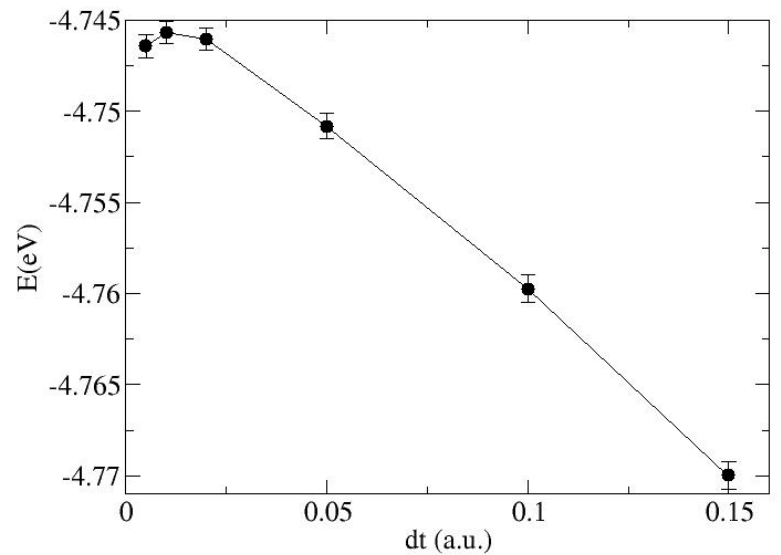

FIG. 3: Dots: diffusion Monte Carlo total energy for the $\mathrm{Mg}$ atom (left panel) and binding energy of the $\mathrm{H}_{2}$ molecule (right panel) as function of time step. Calculations have been performed with the locality approximation. Square: calculation performed with the scheme proposed by Casula 29 .

Mg bulk

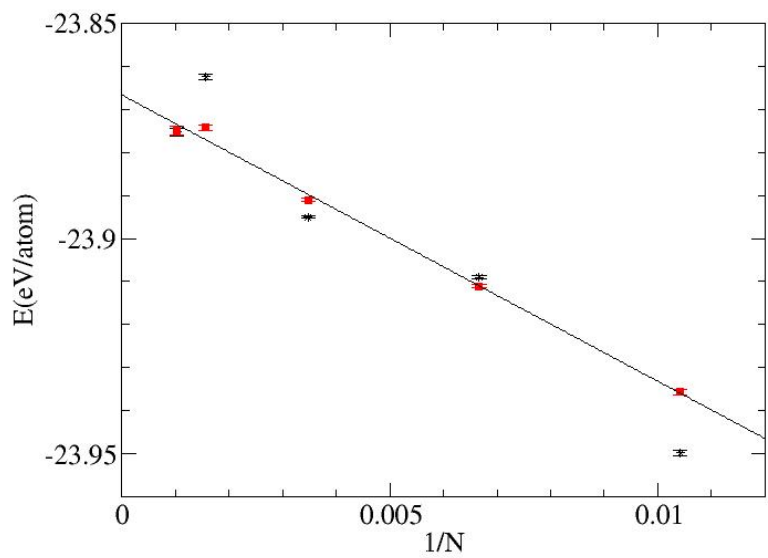

FIG. 4: Diffusion Monte Carlo total energy for the $\mathrm{Mg}$ crystal as function of $1 / N$, where $\mathrm{N}$ is the number of particles in the simulation cell. Stars and squares correspond to raw and DFT corrected (see text) results, solid line is a linear least square fit to the DFT corrected results. 


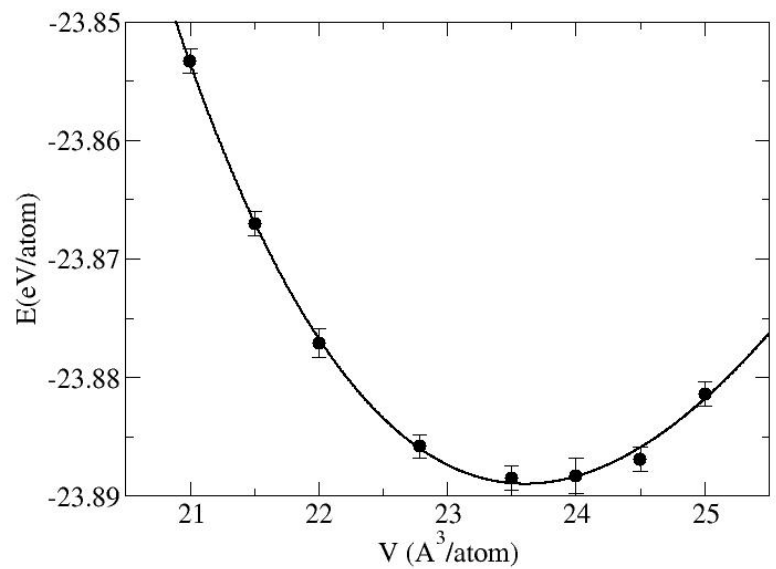

FIG. 5: Diffusion Monte Carlo free energies at $298 \mathrm{~K}$ for the $\mathrm{Mg}$ crystal as function of volume $V$. Dots correspond to DMC calculations extrapolated to infinite size, and include vibrational free energies calculated with DFT-PBE. Solid line is a least squares fit to a Birch-Murnaghan equation of state.

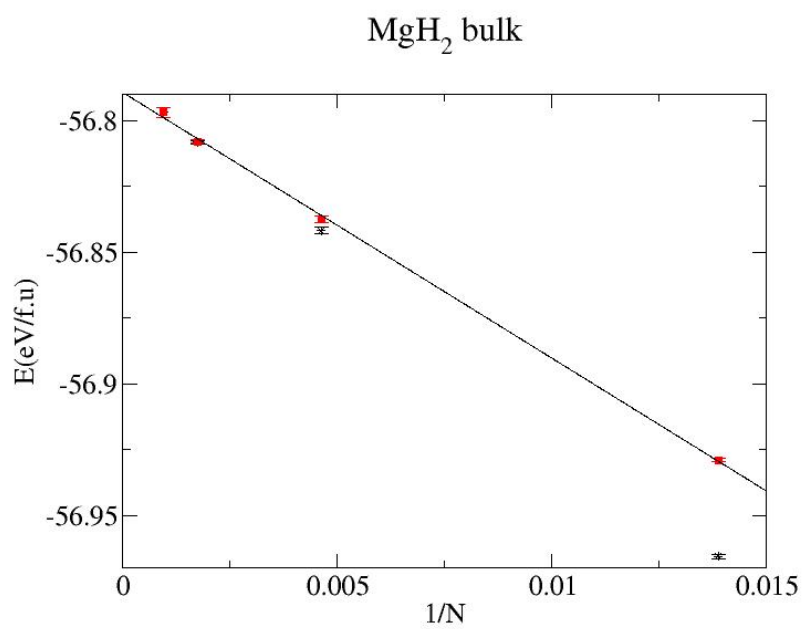

FIG. 6: Diffusion Monte Carlo total energy for the $\mathrm{MgH}_{2}$ crystal as function of $1 / N$, where $\mathrm{N}$ is the number of particles in the simulation cell. Stars and squares correspond to raw and DFT corrected (see text) results, solid line is a linear least square fit to the DFT corrected results. 


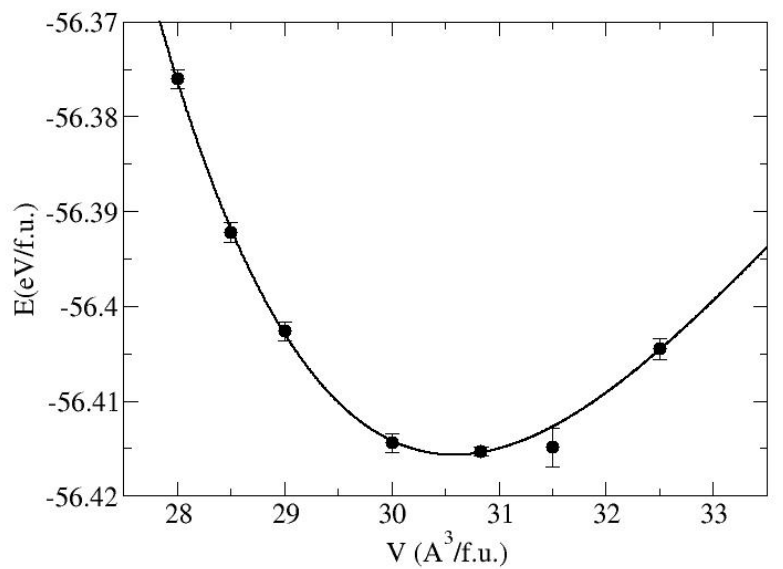

FIG. 7: Diffusion Monte Carlo free energies at $260 \mathrm{~K}$ for the $\mathrm{MgH}_{2}$ crystal as function of volume $V$. Dots correspond to DMC calculations extrapolated to infinite size, and include vibrational free energies calculated with DFT-PBE. Solid line is a least squares fit to a Birch-Murnaghan equation of state. 